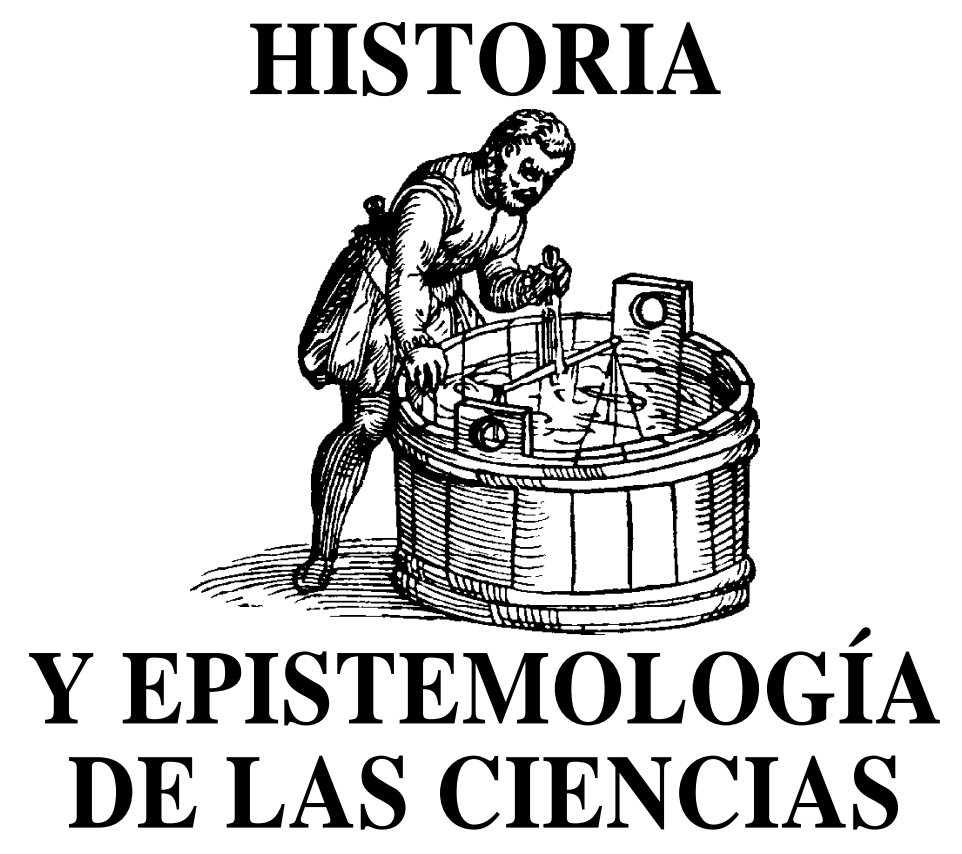

\title{
REFLEXIONES SOBRE LA CIENCIA Y LA EPISTEMOLOGÍA CIENTÍFICA
}

GALLEGOS, JOSÉ A.

Departamento de Didáctica de Ciencias Experimentales. Facultad de Ciencias de la Educación. Universidad de Granada. Polígono Universitario Cartuja. 18071 Granada.

\begin{abstract}
SUMMARY
From the epistemologic point of view, some constructivist trends seem to border on a extreme idealism of kantian overtones; they suggest that the natural world has no existence apart from what the individual creates in his mind. On the other hand, from the methodological point of view, sometimes a valid approach is excessively privileged (such as the fact that the interpretation of observation is always done within a previous theoretical framework) or the didactic effectiveness of some specific approaches is highlighted (such as the usefulness of the History of Science for the learning of some concepts and scientific theories). In both cases perhaps the importance of induction is underestimated, or the essential part of the scientific method is rejected, or the advantages of some other alternatives are forgotten. This paper offers some reflections on the convenience of adopting a middle position instead of leaning to these two different points.
\end{abstract}




\section{INTRODUCCIÓN}

Algunos trabajos sobre facetas epistemológicas o metodológicas con enfoque constructivista del proceso de enseñanza-aprendizaje contienen, a veces, afirmaciones o juicios de valor que dan la impresión de volver a situarse en la línea del constructivismo más radical (de corte totalmente idealista) que ya se había abandonado; también parecen encerrar un desprecio total hacia el inductivismo (que supera con mucho la postura aristotélica respecto de él) y crean la impresión de reclamar una exclusividad metodológica (en relación con el uso de la historia de la ciencia en el caso de Solves y Traver, 1996) que ya parecía superada.

Porque, si se parte del supuesto de que las personas construyen sus ideas sobre cómo funciona el mundo a partir de las observaciones, experiencias y conocimientos acumulados, y que cada individuo extrae sus consecuencias sobre dicho funcionamiento de forma diferente a cómo puede hacerlo otro individuo, surge una corriente de estructuración de la enseñanza que se ha dado en llamar constructivismo. El constructivismo tiene un matiz de oposición al positivismo empirista en tanto en cuanto éste supone que el mundo tiene su propia realidad y el aprendizaje consiste en tratar de conocer esa realidad. Por el contrario, «el constructivismo, especialmente el constructivismo radical no busca una descripción de la verdad ni suscribe la idea de que en la investigación se progresa hacia la verdad» (Novak, 1988, p. 219). En consecuencia con ese marcado tinte idealista, el «constructivismo radical» considera inútiles todos los esfuerzos por conocer el mundo tal como es y afirma que nunca llegaremos a conocer una realidad exterior a nosotros, puesto que todo el conocimiento es subjetivo.

Uno de los autores que más ha trabajado en esta línea (Novak, 1988) expresa las ideas fundamentales con estas palabras: «el constructivismo humano es un esfuerzo por integrar la psicología del aprendizaje humano y la epistemología de la construcción de conocimientos (p. 220) y pone el énfasis en que tanto en la psicología como en la epistemología debemos centrarnos en el proceso de fabricación de significados que supone adquisición o modificación de conceptos y relaciones entre conceptos» (p. 220).

Por lo tanto:

«Crear nuevos conocimientos es, por parte del creador, una forma de aprendizaje significativo. Ello supone a veces, el reconocimiento de nuevas regularidades en hechos u objetos, la invención de nuevos o la extensión de los viejos conceptos, el reconocimiento de nuevas relaciones (proposiciones) entre conceptos y, en los saltos más creativos, la reestructuración importante de estructuras conceptuales para ver nuevas relaciones de orden superior. Estos procesos pueden ser concebidos como parte del proceso del aprendizaje asimilativo, que supone la adición de nuevos conceptos, la diferenciación progresiva de los ya existentes, el aprendizaje supraordenado (en ocasiones) y nuevas e importantes reconci- liaciones integradoras entre las estructuras conceptuales» (pp. 219-220).

Otro aspecto crucial que aporta el constructivismo es la reflexión de que el carácter objetivo, libre de valores, de la ciencia o de otros campos de creación del conocimiento es sólo «un mito del positivismo mantenido a costa de ignorar las miríadas de decisiones subjetivas y basadas en valores que cualquier persona implicada en la producción de conocimientos debe hacer» y que es la «integración constructiva de pensamiento, sentimiento y acción la que da un carácter distintivamente humano a la producción de conocimientos» (Novak, 1988, p. 221). En ese sentido, parece deducirse que el conocimiento científico tiene tanto de construcción personal y social - producto del esfuerzo colectivo de la humanidad-que corre el peligro de ser bastante o totalmente subjetivo.

En tercer lugar, las corrientes constructivistas consideran a la persona que aprende como una parte activa e interesada en el proceso de aprendizaje y sostiene que sus conocimientos previos juegan un papel decisivo cuando se construyen los nuevos significados; los que aprenden van construyendo una concepción del mundo cada vez más cercana al modelo que acepta la comunidad científica. El alumno llega a la clase con unas ideas previas y el profesor debe tener en cuenta estas ideas, puesto que influyen en el significado que los alumnos dan a las situaciones que se les presentan.

A pesar de los esfuerzos de algunos teóricos por conseguirlo, todo este conjunto de corrientes, que no constituyen un modelo único ni unitario, tampoco constituyen un cuerpo de doctrina teórico sólido, sobresaliendo por su pragmatismo. Sus fundamentos teóricos hay que buscarlos en las conclusiones de Piaget (1954, 1970, 1975), en los planteamientos de Ausubel (1968), Ausubel y otros (1986) y, más remotamente, en Kuhn (1971) (en relación con que el cambio de conocimiento puede significar un cambio de paradigma desde el punto de vista personal). Del primero considera la adquisición del conocimiento por construcción, siguiendo las pautas de adaptación y equilibración (asimilación y acomodación); del segundo toma la importancia que debe darse a las ideas previas de los que aprenden como punto de partida. Ahora bien, si, para Piaget, lo que se construyen son las estructuras operatorias, para los constructivistas, lo que se construye son los significados de los contenidos (López, 1989). Por ello, si, en el cognitivismo, el desarrollo consiste en avanzar en las estructuras operatorias, en el constructivismo, consiste en una aproximación entre las ideas de los alumnos y las ideas de los científicos.

En estos últimos años, ha pasado ya el entusiasmo inicial, quizá relacionado con una hipervaloración de los aspectos novedosos o de la consideración como novedosos de aspectos ya desde antiguo juzgados como significativos para el aprendizaje (Gallegos, 1996). Las posturas se han diversificado y han empezado a aparecer análisis críticos más matizados para unos u otros aspectos del «complejo constructivista», tanto desde el punto 
de vista epistemológico (Driver y Oldham, 1986; Mathews, 1989; Suchting, 1992; Matthews, 1994b) como desde el más pragmático de la metodología y la actuación en el aula (Garrison y Bentley, 1990; Coll, 1991, 1994).

En esta nota, con la exclusiva finalidad de sugerir la conveniencia de reconsiderar posturas o enfoques como los mencionados al comienzo, y sin el más mínimo ánimo de polémica, me limito a ofrecer ciertas consideraciones sobre esos aspectos.

\section{ALGUNAS REFLEXIONES EPISTEMOLÓ- GICAS Y METODOLOGICAS}

\section{Sobre la realidad del mundo y la posibilidad humana de conocerlo}

Sin pretender resolver un problema fundamental y permanente de la ontología y la cosmología desde la Grecia clásica, sólo quiero modestamente llamar la atención sobre una premisa básica para el científico: existe un mundo objetivo exterior al estudioso y éste se aplica a conocer cómo es.

No se trata, simplemente, de que el científico deje de lado la discusión de la existencia real del mundo y actúe «como si existiera realmente». En esa postura psicológica, no parece fácil explicar que haya habido, y siga habiendo, tantas personas que dedican su vida a estudiar, analizar y tratar de descubrir cómo es un mundo que podría no ser más que una mera entelequia intelectual humana. Es que realmente se da por supuesto que el mundo tiene una existencia propia independiente del observador que lo analiza, como ya Siegel (1989) sostiene explícitamente. Y la afirmación anterior no impide que el científico tenga que aceptar que su intervención en la observación y análisis de la realidad pueden interferir en el funcionamiento del mundo cuya dinámica pretende analizar. Esta constatación se hace especialmente candente en muchas investigaciones ecológicas (porque el hombre es un componente del sistema mismo que analiza) y en algunos experimentos en la esfera de la dinámica atómica desde la perspectiva cuántica, que proporcionaron la base para el enunciado del principio de incertidumbre de Heisemberg.

Por otra parte, también la ciencia da como indiscutible que el hombre tiene una mínima capacidad para comprender el mundo o, al menos, ir comprendiendo progresivamente el mundo; en caso contrario, también sería totalmente absurdo el esfuerzo por intentarlo (Bunge, 1975; Koyré, 1977). El científico ni siquiera entra en la discusión de los sofistas antiguos con los que se enfrentó Sócrates (Platón, $400 \mathrm{aC}$ ), ni discute con Kant (1787) -como hacen Herbart y Bolzano en Alemania, Balmes (1845) en España, y otros- la capacidad de la mente humana para comprender las cosas; adopta en este campo una postura totalmente antiescéptica, como ya hizo Aristóteles. Eso no quiere decir que no sea consciente de las limitaciones de su intelecto, aunque a veces se com- porte como un «realista ingenuo». Cuando reflexiona sobre su propia ciencia, sabe muy bien que su acercamiento al mundo que le rodea necesariamente tiene que proceder por aproximaciones sucesivas. Por ello sabe (aunque a veces parece olvidarlo lastimosamente) que sus descripciones del mundo son provisionales y deben seguir siendo revisadas y refinadas, quizá indefinidamente (Artigas, 1989).

\section{Sobre la inducción}

Desde el siglo XVIII parece ser de dominio común que el método científico es eminentemente inductivo y experimental, y ello ha dado lugar a desarrollos conceptuales muy importantes y significativos (Hume, 1977; Russell, 1975; Mill, 1961; Carnap, 1962; Ayer, 1965; Salmon, 1975; Popper, 1974). Pero, cuando se ha reflexionado más detenidamente sobre el quehacer real de los científicos, ya no se ha visto tan claro que el esquema tradicional (observación, hipótesis explicativas, comprobación, teoría) sea tan unidireccional, intelectualmente libre de «pre-juicios» y epistemológicamente fuente única de conocimientos (Popper, 1967; Medawar, 1969; Polanyi, 1973; Lakatos y Musgrave, 1975; Garrison, 1986, entre otros). Todo ello ha llevado a desterrar la perspectiva del «inductivismo ingenuo», tal como lo analiza y critica Chalmers (1988).

No obstante, parece necesario aceptar que, de alguna manera, se habrá llegado a elaborar el cuadro teórico existente. Si hay una teoría que dirige y dentro de la cual se interpretan las observaciones que se llevan a cabo y los hechos que ocurren, que prepara y estructura los experimentos a realizar para comprobar las hipótesis propuestas, ha debido ser consecuencia de un proceso previo de observación, estudio y estructuración mental. $\mathrm{Y}$ en este terreno no parece que se haya superado ni cambiado, en lo esencial, lo que ya Aristóteles (330 aC) comprendió hace veintitres siglos y discute ampliamente en su «analítica». Efectivamente, él da mayor valor al silogismo deductivo porque lo aprecia como lógicamente más consistente, pero reconoce que los enunciados universales sólo se consiguen tras una detallada y abundante acumulación de informaciones parciales (concretas), que denomina silogismo por inducción. Justamente, la duda formal que puede quedar como consecuencia de una generalización por inducción es, precisamente, la dificultad (¿imposibilidad demasiadas veces?) de tener en cuenta la «totalidad de los casos particulares». Espigo algunas frases significativas que ilustran estas ideas:

«La inducción o razonamiento inductivo consiste en establecer una relación entre un término extremo y el término medio, a través de otro extremo... Con todo, debemos entender por $C$ la suma de todos los casos particulares, pues la inducción se realiza teniendo en cuenta la totalidad de éstos... Donde hay término medio, el silogismo se hace a través del término medio; donde no lo hay, procede por inducción. Éste es el sentido en que la inducción se opone al silogismo... Así pues, por naturaleza, el silogismo, al proceder a través del término medio, es anterior y más cognoscible, mientras 
que el silogismo por inducción nos resulta más claro a nosotros» (Anal. Prim. II, 23, p. 346).

Entre los muchos filósofos medievales que discuten la difícil «cuestión de los universales», me complace recordar especialmente al olvidado Roger Bacon (1280), que introduce ya la exigencia metodológica de la «experimentación» (en sentido moderno) para adquirir un «conocimiento cierto» de las cosas. Su nuevo enfoque resultó tan revolucionario para su tiempo que no fue valorado en absoluto (más bien fue considerado negativamente) y condujo al olvido de su obra, hasta que su homónimo Francisco Bacon (1620) lo actualizará, casi cuatro siglos más tarde, con un lenguaje nuevo, en un esquema teórico más acabado $\mathrm{y}$, sobre todo, en un momento cultural totalmente distinto.

En la misma línea aristotélica se sitúa Tomás de Aquino (1270), que defiende que la naturaleza de la piedra la consideramos siempre en una piedra particular; la naturaleza del caballo, en caballos particulares... Así es como nuestro entendimiento conoce siempre el universo partiendo de lo particular; pero lo particular sólo es aprehensible por el conocimiento sensible. Consiguientemente, estamos siempre necesitados de la experiencia sensible, y todo ello tiene que investigarse, ineludiblemente, en el espacio y en el tiempo (Sum. Th. I, 84, p. 5).

Por lo tanto, habrá que recuperar de nuevo un cierto distanciamiento crítico respecto de la importancia de la inducción en el conjunto de los procesos mentales para adquirir conocimiento sobre el mundo que nos rodea; no se puede olvidar el alcance puramente probabilístico (aproximativo) de los conocimientos basados en la inducción. Pero eso no parece que pueda implicar, en modo alguno, ni su olvido, ni siquiera su menosprecio en el proceso de aprendizaje. Será, seguramente, cierto, que el alumno utiliza sus «ideas previas» para interpretar su entorno (aunque no en mayor medida que el profesor utiliza sus esquemas mentales y sus teorías, que son suyas en tanto en cuanto manifiestan «su comprensión» de las teorías aprendidas en el conjunto de la ciencia). Pero de ahí no se puede concluir el que esas ideas previas no sean consecuencia de una cierta interpretación de las observaciones, de un establecimiento de relaciones causales (más o menos justificadas) entre factores concomitantes, etc. Será necesario, pues, seguir teniendo en cuenta esta forma en la que la mente va adquiriendo conocimiento y enfocarla dentro de unos parámetros más rigurosos, con unos criterios de «comprobación de causalidad» más estrictos, etc. Una matizada discusión sobre la observación de la realidad y la «idealización» científica llevada a cabo por Galileo y Newton en la que se mueve la física actual, sobre todo, se puede encontrar en Matthews (1994b, pp. 262-264).

\section{Sobre la historia de la ciencia y el método científico}

Apoyarse en la historia de las ciencias para transmitir la idea de que la ciencia es una construcción de conocimientos para resolver problemas es una metodología útil y fructífera si se maneja adecuadamente. No es necesario insistir excesivamente en este aspecto, que ya ha sido tratado abundantemente (Koyré, 1977; Gagliardi, 1988; Nielsen y Thomsen, 1990; Izquierdo, 1994) y que Matthews (1994b) ha resumido muy acertadamente.

Pero parece excesivo afirmar que sea la única forma de conseguirlo o que «la imagen de la ciencia que se transmite en la universidad a los futuros profesores -como unos contenidos ya hechos, como una serie de leyes que se deducen lógicamente a partir de unos principios- es, si no la razón fundamental, sí al menos una razón preponderante de distorsión en la comprensión de la ciencia (Solbes y Traver, 1996, p. 104). Yo, personalmente, estimo que caben otras alternativas para no caer en ese enfoque, que me parece ciertamente deformado y deformante. Sin embargo, muchos de los profesores actualmente en ejercicio no tuvimos demasiadas ocasiones de conocer la historia de la ciencia durante nuestros estudios universitarios, y no por ello parece que acabáramos con una visión tan distorsionada de las teorías científicas ni del quehacer científico.

Además, surge a veces la duda de si no se está entrando en cierta contradicción mental cuando se critica que la ciencia se presenta como una serie de leyes que se deducen lógicamente de unos principios, poco después de haber afirmado que es una deformación seguir manteniendo que los conocimientos científicos se forman por inducción a partir de las observaciones y los experimentos.

\section{Sobre los genios y la originalidad de su pensamiento}

Presentar como opinión la propia convicción de que los grandes genios no han colaborado en mayor medida que todos los muchos hombres y mujeres que han trabajado previamente en un campo concreto de la ciencia es totalmente respetable (Solbes y Traver, 1996, p. 104), pero no parece posible aceptar que Newton, o Darwin, o Maxwell, o Einstein, o Wegener, por ejemplo, no tuvieron una participación decisiva en ese trabajo de conjunto. Todos los científicos de su tiempo tenían los mismos datos disponibles, y no todos fueron capaces de enfocarlos, reestructurarlos o reinterpretarlos de la misma forma.

Por otra parte, tampoco parece que podamos despreciar lo que yo vengo denominando desde hace tiempo efecto eureka, recogiendo un aspecto quizá legendario pero psicológicamente muy consistente que Vitrubio contaba de Arquímedes (seguramente el mismo que Gardner llama efecto ajá). Sería muy interesante que los psicólogos le dedicaran más atención (no he conseguido encontrar trabajos psicológicos en esta línea) y nos ilustraran detalladamente cómo se dan esas asociaciones de ideas en la mente, que permiten enfocar desde nuevas perspectivas situaciones conocidas (con un pensamiento típicamente divergente, más creativo). No obstante, tenemos ciertos datos y recopilaciones en esa línea, que son sugerentes y que convendría no olvidar (Nicolle, 1932; Taton, 1967; Torrance, 1969). 
Resulta especialmente oportuno aquí llamar la atención sobre el eco que se hace Kuhn (1971) de ciertas experiencias de los investigadores cuando dice que «los científicos hablan con frecuencia de las "vendas que se les caen de los ojos" o de la "iluminación repentina" que "inundó" un enigma previamente oscuro, y eso hace que sus componentes se vean de una nueva manera que permita por primera vez resolverlos. En otras ocasiones, la iluminación pertinente se presenta por la noche». En esa misma línea se sitúan las cuatro etapas de la «iluminación científica» (preparación, incubación, iluminación, verificación) que estructura Butcher (1974).

\section{Sobre el papel del azar en los descubrimientos cien- tíficos}

Para terminar, quiero comentar que quizá se haya exagerado (incluso excesivamente) la influencia del azar en los descubrimientos científicos o se haya achacado a la inspiración lo que corresponde, al menos en parte, al trabajo paciente y tenaz. Pero no parece que estemos autorizados a negar totalmente la importancia del primero, tanto en el descubrimiento de los rayos X por parte de Roentgen, como en el descubrimiento de la radiactividad por parte de Becquerel (que son quizá dos de los ejemplos recientes más usados para ilustrar esta idea), o el de la lisozima por Fleming (1929). Es más, aunque leamos con un espíritu fuertemente crítico la historia de los veinte experimentos que, según Harré (1986), han cambiado nuestra visión del mundo y aunque minimicemos las conclusiones a que llega Taton (1967), no parece que podamos eliminar el efecto de la «casualidad» en todos los ejemplos recogidos en este último trabajo.

Desde un punto de vista más filosófico, además, se podría plantear la discusión de si es posible eliminar el

\section{REFERENCIAS BIBLIOGRÁFICAS}

AGUIRRE, A. (1935). La ciencia positiva en el siglo XIII: Rogerio Bacon. Barcelona: Labor.

ARISTÓTELES (330 aC). Analítica primera. Ed. Samaranch, F. 1973. Madrid: Aguilar.

ARTIGAS, M. (1989). Filosofía de la ciencia experimental. Pamplona: Eunsa.

AUSUBEL, D. (1968). Educational Psychology: a cognitive view. Nueva York: Grune and Stratton.

AUSUBEL, D.P., NOVAK, J.D. y HANESIAN, H. (1986). Psicología educativa. Un punto de vista cognoscitivo. México: Trillas.

AYER, A.J. (1965). El positivismo lógico. México: Fondo de Cultura Económica. azar en el proceso de investigación, siendo así que la casualidad parece ser un constitutivo esencial de la trama de lo real. De todas formas, el descubrimiento científico propiamente dicho sí que parece residir precisamente en la capacidad de interpretar y reproducir las series causales de fenómenos que han «coincidido» en un determinado momento.

\section{CONCLUSIÓN}

De las reflexiones anteriores, quizá se puedan extraer dos conclusiones:

- La primera, que, metodológicamente, podremos (y deberemos) aprovechar al máximo, en clase, todo tipo de recursos, técnicas y tendencias didácticas; pero puede que sea imprescindible que tengan que estar encajadas en un cuadro intelectualmente coherente, para que no originen distorsiones ni dificultades de comprensión en los alumnos (en la línea que Laburu [1996] ha señalado).

- La segunda, insistir en la propia toma de conciencia de lo parciales y provisionales que siguen siendo nuestros conocimientos y, en consecuencia, seguir haciendo un ejercicio de modestia. Por un lado, reconociendo las aportaciones de todos los que nos han precedido $\mathrm{y}$ aprovechando los atisbos de comprensión de la realidad que tuvieron. Por otro, trabajando ilusionadamente en el campo de la didáctica y aportando nuestra colaboración, pero con la conciencia muy clara de que habrá que seguir haciendo un esfuerzo ímprobo por equilibrar las distintas tendencias sin desplazarse unilateralmente hacia ninguna, sino aprovechando lo que haya de didácticamente valioso en todas ellas.
BACON, F. (1620). Novum Organum. Edición Enciclopedia Británica. Chicago, 1989.

BACON, R. (1280). Opus Maius (Scientia Experimentalis). (Edición Steele y Delorme (1940): Opera hactenus inedita fratris R. Baconis. París.

BALMES, J. (1845). Obras completas. Madrid: Edición BAC, 1950.

BASKAR, R. (1978). A realist theory of Science. Brighton: Harvester.

BUNGE, M. (1975). Teoría y realidad. Barcelona: Ariel.

BUTCHER, H.J. (1974). La inteligencia humana. Madrid: Marova. 
CARNAP, R. (1962). Logical foundations of probability. Chicago: Chicago Univ. Press.

CHALMERS, A.F. (1984). ¿Qué es esa cosa llamada ciencia? Madrid: Siglo XXI.

COLL, C. (1991). Concepción constructivista y planteamiento curricular. Cuadernos de Pedagogía, 188, pp. 8-11.

COLL, C. et al. (1994). El constructivismo en el aula. Valencia: Graó.

DRIVER, R. y OLDHAM, V. (1986): A Constructivist Approach to Curriculum Development in Science. Studies in Sc. Educ. 13 , pp. 105-122.

FLEMING, A. (1929). On antibacterial action of the cultures of Penicilium with special reference to their use in the insolation of B. influenzae. Brit. J. Exp. Path. 10, pp. 226-236.

GAGLIARDI, R. (1988). Cómo utilizar la historia de las ciencias en la enseñanza de las ciencias. Enseñanza de las Ciencias, 6(3), pp. 291-295.

GALLEGOS, J.A. (1996). Algunos antecedentes del constructivismo. Rev. Educ. Univ. Granada, 9, pp. 277-286.

GARDNER, M. (1986). ¡Ajá! Paradojas. Barcelona: Labor.

GARRISON, J.W. (1986). Some Principles of Postpositivist Philosophy of Science. Educational Researcher, 15(9), pp. 12-18.

GARRISON, J. y BENTLEY, M. (1990). Science Education, Conceptual Change and Breaking with Everiday Experience. Stud. in Phil. and Educ., 10(1), pp. 19-36.

HARRÉ, R. (1986). Grandes exprimentos científicos. Barcelona: Labor.

HUME, D. (1977). Tratado de la naturaleza humana. Madrid: Editora Nacional.

IZQUIERDO, M. (1994). ¿Cómo contribuye la historia de las ciencias en las actitudes del alumnado hacia el aprendizaje de las ciencias? Aula Inv. Educativa, 27, pp. 37-40.

KANT, E. (1787). Crítica de la razón pura. Barcelona: Edición Orbis, 1984.

KOYRÉ, A. (1977). Estudios de historia del pensamiento científico. Madrid: Siglo XXI.

KUHN, D. (1971).La estructura de las revoluciones científicas. México: Fondo de Cultura Económica.

LABURU, C.E. (1996). La crítica en la enseñanza de las ciencias: constructivismo y contradicción. Enseñanza de las Ciencias, 14(1), pp. 93-102.

LAKATOS, I. y MUSGRAVE, A. (comp.) (1975). La crítica y el desarrollo del conocimiento científico. Barcelona: Grijalbo.

LÓPEZ, F. (1989). La exigencia cognitiva en física: interpretación piagetiana e interpretación constructivista. Actas del III Congreso Internacional sobre Didáctica de las Ciencias y la Matemática. Santiago.

MATTHEWS, M.R. (1989). Ernst Mach and Thought Experiments in Science Education. Res. Sc. Education, 18, pp. 251-258.
MATTHEWS, M.R. (1994a). Vino viejo en botellas nuevas: un problema con la epistemología constructivista. Enseñanza de las Ciencias, 12(1), pp. 79-88.

MATTHEWS, M.R. (1994b). Historia, filosofía y enseñanza de las ciencias: la aproximación actual.Enseñanza de las Ciencias, 12(2), pp. 255-277.

MEDAWAR, P. (1969): Induction and intuition in scientific thougth. Londres: Mathuen.

MILL, J.S. (1961). A system of Logic. Madrid: Longman.

NICOLLE, C. (1932). Biologie de l'Invention. París: Alcan.

NIELSEN, H. y THOMSEN, P. (1990). History and Philosophy of Science in the Danish Curiculum. Int. J. of Science Education, 12(3), pp. 308-316.

NOVAK, J.D. (1988). Constructivismo humano: un consenso emergente. Enseñanza de las Ciencias, 6(3), pp. 213-223.

PIAGET, J. (1954). The construction of reality in children. Nueva York: Basic Books.

PIAGET, J. (1970). Piaget's theory, en Mussen (ed.) (1970). Carmichael's manual of child psychology. Nueva York: Wiley.

PIAGET, J. (1975). L'equilibration des structures cognitives. Problème central du devélopment. París: PUF.

PLATÓN (375? aC). Obras completas: El sofista. Madrid: Edición Gredos, 1983-1988.

POLANYI, M. (1973). Personal knowledge. Londres: Routledge y Kegan.

POPPER, K.R. (1967). La lógica de la investigación científica. Madrid: Tecnos.

POPPER, K.R. (1974). Conocimiento objetivo. Madrid: Tecnos.

RUSSELL, B. (1975). Los problemas de la filosofía. Madrid: Labor.

SALMON, W.C. (1975). The foundations of scientific inference. Pittsburg: Pittsburg Univ. Press.

SIEGEL, R. (1989). The rationality of Science, critical thinking and Science Education. Synthese, 80, pp. 9-41.

SOLBES, J. y TRAVER, M.J. (1996). La utilización de la historia de las ciencias en la enseñanza de la física y la química. Enseñanza de las Ciencias, 14(1), pp. 103-112.

SUCHTING, W.A. (1992). Constructivism Deconstructed. Science and Education, 1(3).

TATON, R. (1967). Causalidad y accidentalidad de los descubrimientos científicos. Barcelona: Labor.

TOMÁS DE AQUINO (1270). Summa Theologica I. (Versión BAC [1947] Madrid).

TORRANCE, E.P. (1969). Orientación del talento creativo. Buenos Aires: Troquel.

TOULMIN, S. (1977). La comprensión humana (I): El uso colectivo y la evolución de los conceptos. Madrid: Alianza. 